March 23, 2018 0:19 WSPC/INSTRUCTION FILE paper-ijmpd-form-final

International Journal of Modern Physics D

(C) World Scientific Publishing Company

\title{
SPINNING BOSON STARS AND HAIRY BLACK HOLES WITH NON-MINIMAL COUPLING
}

\author{
Carlos A. R. Herdeiro and Eugen Radu \\ Departamento de Física da Universidade de Aveiro and CIDMA, \\ Campus de Santiago, 3810-183, Aveiro, Portugal \\ herdeiro@ua.pt, eugen.radu@ua.pt \\ Received Day Month Year \\ Revised Day Month Year
}

\begin{abstract}
We obtain spinning boson star solutions and hairy black holes with synchronised hair in the Einstein-Klein-Gordon model, wherein the scalar field is massive, complex and with a non-minimal coupling to the Ricci scalar. The existence of these hairy black holes in this model provides yet another manifestation of the universality of the synchronisation mechanism to endow spinning black holes with hair. We study the variation of the physical properties of the boson stars and hairy black holes with the coupling parameter between the scalar field and the curvature, showing that they are, qualitatively, identical to those in the minimally coupled case. By discussing the conformal transformation to the Einstein frame, we argue that the solutions herein provide new rotating boson star and hairy black hole solutions in the minimally coupled theory, with a particular potential, and that no spherically symmetric hairy black hole solutions exist in the non-minimally coupled theory, under a condition of conformal regularity.
\end{abstract}

Keywords: black holes; scalar fields; no-hair theorems

PACS numbers:

\section{Contents}

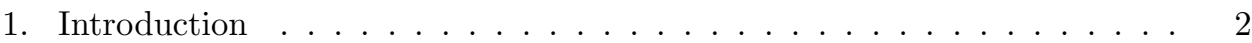

2. The framework ....................... 3

2.1. The action and field equations . . . . . . . . . . 3

2.2. A Smarr relation . . . . . . . . . . . . . . . . . . 3

2.3. The Ansatz and quantities of interest . . . . . . . . . 5

3. Non-minimally coupled boson stars and BHs with scalar hair . . . . . . 6

3.1. Boundary conditions . . . . . . . . . . . . . 6

3.2. Boson star solutions . . . . . . . . . . . . . . . 7

3.3. Black holes with synchronized scalar hair . . . . . . . . . . . 9 9

4. Further remarks . . . . . . . . . . . . . . . . . 11 


\section{Introduction}

Scalar fields play an important role in modern physics, entering a multitude of models ranging from microscopic to cosmic scales. Even though scalar fields are often used as a proxy to model more complex interactions, the detection of the Higgs field shows the existence of fundamental scalar fields! 1

The interaction of scalar fields with gravity is a subject of long-standing interest. Scalar fields are ubiquitous in modelling the physics of the early universe; but there is also an intriguing possibility that such fields could play a role in black hole (BH) physics ${ }^{2[3}$ or even cluster to form smooth horizonless compact objects: the so-called boson stars (BSs) ${ }^{4}$ BSs are held together by their self-generated gravitational field, and are supported against collapse by the dispersive effect due to the wave-like character of the scalar field $!^{5}$ They have been extensively studied in the literature, being considered as possible dynamical astrophysical objects, ${ }^{6}$ black hole mimickers ${ }^{7 / 9}$ or even as a possible constituent of dark matter.10 11] Remarkably, spinning (but not static) BSs possess BH generalizations provided the scalar field co-rotates with the horizon: BHs with synchronised hair!12]13

It is therefore worthwhile studying more general couplings (than minimal coupling) of scalar fields to gravity. Restricting to a single complex scalar field $\Psi$, perhaps the simplest and best motivated extension is the inclusion of an explicit coupling between $\Psi$ and the Ricci scalar curvature of the spacetime, $R$, of the form $\xi \Psi^{*} \Psi R$, where $\xi$ is a dimensionless coupling constant and $\Psi^{*}$ denotes the complex conjugate field. There are reasons to believe that such a nonminimal coupling term (as well as other couplings) appears naturally ${ }^{14}[16$ For example, a nonminimal coupling is generated by quantum corrections even if it is absent in the classical action and it is required in order to renormalize the theory ${ }^{17}$ At a fundamental level, it is not known if there is a preferential value of $\xi$; however, two cases occur most frequently in the literature: "minimal coupling" $(\xi=0)$ and "conformal coupling" $(\xi=1 / 6)$. The latter name is justified by the fact that conformal invariance of the model dictates $\xi=1 / 6$ for a massless scalar field $!^{17}$

In many physical situations, the inclusion of a $\xi \neq 0$ term leads to new interesting physical effects even at the classical level. Well known examples include the inflationary scenario with a nonminimally coupled "inflaton" field ${ }^{18}$ and the Bronnikov-Melnikov-Bacharova-Bekenstein (BMBB) BH with conformal scalar hain] ${ }^{19}\left[20\right.$ More recent studies have considered e.g. traversible wormholes, ${ }^{25 \mid 26}$ solitons and BHs with a non-minimally coupled gauged Higgs field,,$\sqrt[27]{29}$ as discussed elsewhere. ${ }^{14}[16$

In this context, it is worthwhile investigating how a non-minimal coupling affects the properties of BHs with synchronised scalar hair together with their solitonic limit - spinning BSs. So far, only the spherically symmetric limit has been

${ }^{a}$ We remark that the BMBM BH is rather special. The spherically symmetric asymptotically flat BHs with generic $\xi$ cannot support nonminimally coupled spatially regular neutral scalar fields 2124 
investigated, $, 30,33$ in which case only solitonic solutions are possible. In this paper we report spinning BHs and $\mathrm{BH}$ solutions with synchronised hair of the Einstein(complex, massive) Klein-Gordon equations with a nonminimal coupling $\xi \Psi^{*} \Psi R$, extending previous results $\$ \frac{12 \sqrt{35}}{}$ to this case.

\section{The framework}

\subsection{The action and field equations}

We shall be working with an Einstein-Klein-Gordon (EKG) field theory, describing a massive complex scalar field $\Psi$ with a non-minimally coupling to Einstein's gravity,

$$
S=\int d^{4} x \sqrt{-g}\left[\frac{1}{16 \pi G} R-\frac{1}{2} g^{a b}\left(\Psi_{, a}^{*} \Psi_{, b}+\Psi_{, b}^{*} \Psi, a\right)-\mu^{2} \Psi^{*} \Psi-\xi \Psi^{*} \Psi R\right],
$$

where $G$ is Newton's constant, $\mu$ is the scalar field's mass and $\xi$ is a dimensionless constant.

The equations of the model are

$$
\begin{aligned}
& R_{a b}-\frac{1}{2} g_{a b} R=8 \pi G T_{a b}^{(e f f)} \\
& \nabla^{2} \Psi=\left(\mu^{2}+\xi R\right) \Psi
\end{aligned}
$$

where we define the effective energy-momentum tensor,

$$
T_{a b}^{(e f f)}=T_{a b}^{(\psi)}+T_{a b}^{(\xi)}
$$

which is the sum of two different contributions

$$
\begin{aligned}
T_{a b}^{(\psi)} & =\Psi_{, a}^{*} \Psi_{, b}+\Psi_{, b}^{*} \Psi_{, a}-g_{a b}\left[\frac{1}{2} g^{c d}\left(\Psi_{, c}^{*} \Psi_{, d}+\Psi_{, d}^{*} \Psi_{, c}\right)+\mu^{2} \Psi^{*} \Psi\right], \\
T_{a b}^{(\xi)} & =2 \xi\left[\left(R_{a b}-\frac{1}{2} g_{a b} R\right) \Psi^{*} \Psi+g_{a b} \nabla^{2}\left(\Psi^{*} \Psi\right)-\left(\Psi^{*} \Psi\right)_{; a b}\right] .
\end{aligned}
$$

It is useful to define an "effective" Newton's constant

$$
\frac{1}{G_{e f f}} \equiv \frac{1}{G}-32 \pi \xi \Psi^{*} \Psi
$$

\subsection{A Smarr relation}

We are interested in stationary, axisymmetric, asymptotically flat BH spacetimes. These geometries possess: 1) a unique translational Killing vector $K^{a}$ which is timelike and normalized to $K^{a} K_{a}=-1$ near spatial infinity; 2) a unique rotational Killing vector $\tilde{K}^{a}$ normalized by demanding that its orbits to be closed curves with parameter length $2 \pi$. Then, a null vector $\chi$ exists that is tangent to the horizon null generator; it can be expressed as

$$
\chi=K+\Omega_{H} \tilde{K},
$$


where $\Omega_{H}$ is the angular velocity of the BH. In general both $K$ and $\tilde{K}$ act nontrivially on the scalar $\Psi$, and only the energy-momentum tensor remains invariant (in particular $\left.K \Psi^{*} \Psi=\tilde{K} \Psi^{*} \Psi=0\right)$.

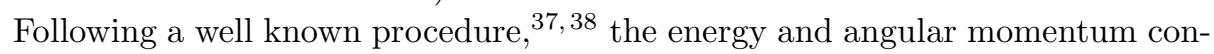
tent of the spacetime may be split into different components. One starts with the identities

$$
M=M_{H}+M_{(\xi)}+M_{(\Psi)}, \quad J=J_{H}+J_{(\xi)}+J_{(\Psi)},
$$

where $M$ and $J$ are the ADM mass and total angular momentum, and

$$
M_{H}=-\frac{1}{4 \pi G} \oint_{H} d S_{a b} \nabla^{a} K^{b}, \quad J_{H}=\frac{1}{16 \pi G} \oint_{H} d S_{a b} \nabla^{a} \tilde{K}^{b},
$$

are the horizon mass and angular momentum, computed as Komar integrals on a spatial section of the horizon. The matter contributions are given by 3 -volume integrals that read

$$
\begin{array}{r}
M_{(\xi),(\Psi)}=-2 \int_{\Sigma} d S_{a}\left(T_{b}^{(\xi),(\Psi) a} K^{b}-\frac{1}{2} T^{(\xi),(\Psi)} K^{a}\right) \\
J_{(\xi),(\Psi)}=\int_{\Sigma} d S_{a}\left(T_{b}^{(\xi),(\Psi) a} \tilde{K}^{b}-\frac{1}{2} T^{(\xi),(\Psi)} \tilde{K}^{a}\right) .
\end{array}
$$

Remarkably, for regular configurations with an asymptotically vanishing scalar field, a straightforward computation shows that the non-minimal coupling contribution to both $M$ and $J$ can be expressed as a total divergence, the only non-vanishing contribution being the horizon surface integral,

$$
M_{(\xi)}=2 \xi \oint_{H} d S_{a b} \Psi^{*} \Psi \nabla^{a} K^{b}, \quad J_{(\xi)}=-\xi \oint_{H} d S_{a b} \Psi^{*} \Psi \nabla^{a} \tilde{K}^{b} .
$$

As usual, ${ }^{[37}$ one can express $d S_{a b}$ as $\chi_{[a} n_{b]} d A$, where $n_{a}$ is the other null vector field orthogonal to the horizon, normalized as $n_{a} \chi^{a}=-1$, and $d A$ is the surface area element of the horizon. After replacing relations (10) and (12) into (9), one arrives at the following Smarr mass formula which relates the temperature, entropy and the global charges

$$
M=2 T_{H} S+2 \Omega_{H}\left(J-J_{(\Psi)}\right)+M_{(\Psi)} .
$$

Here, $T_{H}=\kappa / 2 \pi$ is the Hawking temperature of the BHs (where $\kappa$ is the surface gravity, defined as usual in terms of $\chi$ ), while $M_{(\Psi)}$, and $J_{(\Psi)}$, are the scalar field energy and angular momentum outside the $\mathrm{BH}$, given by (11).

The entropy $S$ of the BHs has an extra contribution with respect to that in Einstein's gravity, due to the non-minimal coupling with the scalar field

$$
S=S_{E}+S_{(\xi)}, \quad \text { with } S_{E}=\frac{1}{4 G} \int_{H} d A, \quad S_{(\xi)}=-4 \pi \xi \int_{H} \Psi^{*} \Psi d A,
$$


a relation which can also be written in the suggestive formb

$$
S=\frac{1}{4} \int_{H} \frac{1}{G_{e f f}} d A,
$$

where $G_{e f f}$ is the effective Newton's constant given by (77).

The solutions should also satisfy the first law of $\mathrm{BH}$ thermodynamics:

$$
d M=T_{H} d S+\Omega_{H} d J .
$$

\subsection{The Ansatz and quantities of interest}

The non-minimally coupled BSs and hairy BHs (HBHs) are constructed with the same ansatz used in previous works ${ }^{[12] 13}$ Working in a coordinate system with $K=\partial_{t}$ and $\tilde{K}=\partial_{\varphi}$, we consider a line element

$$
\begin{array}{r}
d s^{2}=e^{2 F_{1}}\left(\frac{d r^{2}}{N}+r^{2} d \theta^{2}\right)+e^{2 F_{2}} r^{2} \sin ^{2} \theta(d \varphi-W d t)^{2}-e^{2 F_{0}} N d t^{2}, \\
\text { with } N \equiv 1-\frac{r_{H}}{r},
\end{array}
$$

and a scalar field

$$
\Psi=\phi(r, \theta) e^{i(m \varphi-w t)} .
$$

Consequently, the problem contains five unknown functions $\left\{F_{i}, W ; \phi\right\}$ which depend on $(r, \theta)$ only. Also, $w$ is the scalar field frequency and $m= \pm 1, \pm 2 \ldots$ is the azimuthal harmonic index. Without loss of generality, we take $w>0$.

The BHs have a horizon located at $r=r_{H}$; the range of $r$ considered in numerics is $r_{H} \leqslant r<\infty$. BSs correspond to the $r_{H}=0$ limit of (17). Most of the quantities of interest are encoded in the expression for the metric functions at the horizon or at infinity. Considering first horizon quantities, the Hawking temperature $T_{H}$, the event horizon area $A_{H}$ and the event horizon velocity $\Omega_{H}$ are

$$
\begin{array}{r}
T_{H}=\frac{1}{4 \pi r_{H}} e^{\left.\left(F_{0}-F_{1}\right)\right|_{r_{H}}}, \quad A_{H}=2 \pi r_{H}^{2} \int_{0}^{\pi} d \theta \sin \theta e^{\left.\left(F_{1}+F_{2}\right)\right|_{r_{H}}}, \\
\Omega_{H}=-\left.\frac{g_{\varphi t}}{g_{t t}}\right|_{r_{H}}=\left.W\right|_{r_{H}} .
\end{array}
$$

The ADM mass $M$ and the angular momentum $J$ are read from the asymptotic sub-leading behaviour of the metric functions:

$$
\begin{array}{r}
g_{t t}=-e^{2 F_{0}} N+e^{2 F_{2}} W^{2} r^{2} \sin ^{2} \theta=-1+\frac{2 G M}{r}+\ldots, \\
g_{\varphi t}=-e^{2 F_{2}} W r^{2} \sin ^{2} \theta=-\frac{2 G J}{r} \sin ^{2} \theta+\ldots
\end{array}
$$

\footnotetext{
${ }^{\mathrm{b}}$ The same expression of entropy is derived by using Wald's formalism $[39,40$
} 
One notices also that the action (1) is invariant under the global $U(1)$ transformation $\Psi \rightarrow e^{i \alpha} \Psi$, where $\alpha$ is constant. Thus, the scalar 4 -current, $j^{a}=$ $-i\left(\Psi^{*} \partial^{a} \Psi-\Psi \partial^{a} \Psi^{*}\right)$, is conserved: $j_{; a}^{a}=0$. It follows that integrating the timelike component of this 4-current in a spacelike slice $\Sigma$ yields a conserved quantity - the Noether charge:

$$
Q=\int_{\Sigma} j^{t} d \Sigma_{t}
$$

with the explicit expression

$$
Q=2 \pi \int_{r_{H}}^{\infty} d r \int_{0}^{\pi} d \theta r^{2} \sin \theta e^{F_{0}+2 F_{1}+F_{2}} \frac{m(w-m W)}{N} \phi^{2} .
$$

The relation expressing the quantisation of the total angular momentum 13

$$
J_{(\Psi)}=m Q
$$

holds for arbitrary values of $\xi$ and can be used to further simplify the Smarr relation (13).

\section{Non-minimally coupled boson stars and BHs with scalar hair}

\subsection{Boundary conditions}

The solutions with $\xi \neq 0$ are constructed by using the same approach as in the minimally coupled case, described at length in our previous work ${ }^{13}$ At spatial infinity, asymptotic flatness dictates

$$
\lim _{r \rightarrow \infty} F_{i}=\lim _{r \rightarrow \infty} W=\lim _{r \rightarrow \infty} \phi=0,
$$

while on the symmetry axis $\theta=0, \pi$ we impose

$$
\partial_{\theta} F_{i}=\partial_{\theta} W=\phi=0 .
$$

The numerical treatment of the problem is simplified by defining a new radial coordinate $x=\sqrt{r^{2}-r_{H}^{2}}$, such that the horizon is located at $x=0$. There we imposec

$$
\left.\partial_{x} F_{i}\right|_{r=r_{H}}=\left.\partial_{x} \phi\right|_{r=r_{H}}=0,\left.\quad W\right|_{r=r_{H}}=\frac{w}{m} .
$$

Note that the horizon boundary condition imposed on $W$ encodes the 'synchronization condition 12

$$
w=m \Omega_{H},
$$

which implies that there is no flux of the scalar field into (or from) the $\mathrm{BH}, \chi^{\mu} \partial_{\mu} \Psi=$ 0 .

The BSs do not possess a horizon and therefore $0 \leqslant r<\infty$. At the origin $(r=0)$ we impose

$$
\partial_{r} F_{i}=\partial_{r} W=\phi=0
$$

${ }^{\mathrm{c}}$ Note that the scalar field does not vanish at the horizon, being a function of $\theta$. 
For any $\xi$, an approximate form of the solution can be constructed on the boundary of the domain of integration, which is compatible with the boundary conditions given above. Also, all solutions reported in this work are symmetric w.r.t. a reflection on the equatorial plane $\theta=\pi / 2$. Moreover, we focus on nodeless solutions (with no zeros of the scalar field in the equatorial plane) since these are typically the most stable ones.

The numerical integration is performed with dimensionless variables introduced by using natural units set by $\mu$ and $G$,

$$
r \rightarrow r / \mu, \quad \phi \rightarrow \phi / \sqrt{4 \pi G}, \quad w \rightarrow w / \mu .
$$

As a result, no dependence on either $G$ or $\mu$ is present in the equations.

\subsection{Boson star solutions}

The minimally coupled spinning BSs $(\xi=0)$ were originally studied, independently, by Schunck and Mielke $e^{41}$ and Yoshida and Eriguchi $\frac{35}{35}$ A relevant more recent work is due to Grandclement, Som and Gourgoulhon. ${ }^{42}$ Turning on the non-minimal coupling, some results of the numerical integration are exhibited in Figs. 1 and 2.

As one can observe from Fig. 1 (top panel), the basic features of the massfrequency diagram found for the minimally coupled solutions are preserved. Firstly, for any coupling, the BSs exist for $w<\mu$; this is a bound state condition which emerges from the long range behavior of the scalar field, where the Ricci scalar tends to zero. As we decrease the frequency, the mass increases until a maximum valued, which is always of the order of $1 / \mu$. Further decreasing $w$ one finds a minimal frequency $w_{\text {min }}$, below which no BS solutions are found. This minimal frequency increases as $\xi$ increases, and decreases for negative values of the coupling constant. Then, for any $\xi$, the BS curve seems to spiral towards a central region of the diagram where numerical accuracy deteriorates. Qualitatively, this is also the behaviour found for spherically symmetric BSs $(m=0)$, in which case, a detailed investigation of the inspiraling behaviour was possible. We have found a similar behaviour for the total angular momentum, $J$, or equivalently, $c f$. (26), for the Noether charge.

As a particular physical property, for any $\xi$, a part of fast spinning BSs possess a toroidal ergo-surface, likewise the minimally coupled case $\stackrel{31}{32}$ In a frequency-mass diagram, this corresponds to the inner part of the spiral starting with a critical configuration marked with a square in Fig. 1] (top panel).

As another physical property, one may wonder how a nonzero $\xi$ affects the compactness of the spinning BSs. Following previous literature ${ }^{50}$ we define the inverse compactness by comparing $R_{99}$, defined as the circumferential radius wherein $99 \%$ of the BS's mass is contained, with the Schwarzschild radius associated to that

${ }^{\mathrm{d}}$ In the spherical case, this maximal value is proportiona $\sqrt{30}$ to $\sqrt{|\xi|}$. For spinning solutions, however, the numerical accuracy deteriorates before large enough values of $|\xi|$ can be considered; thus a similar relation could not be derived. 

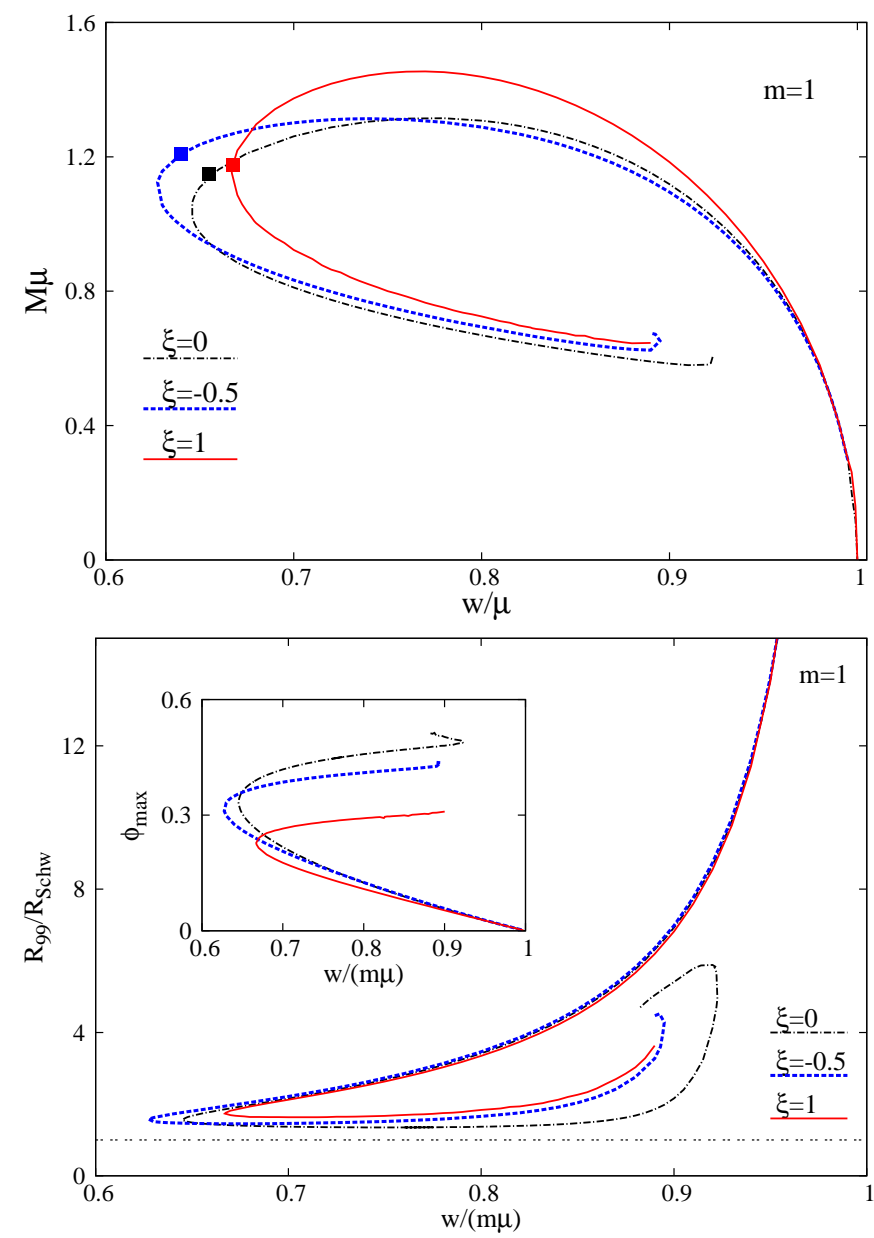

Fig. 1. (Top panel) The frequency-mass diagram is shown for families of spinning boson stars with $m=1$ and three different values of the coupling constant $\xi$. The points highlighted with a square indicate the appearance of an ergo-region (an ergo-torus) when moving towards the centre of the spiral. (Bottom panel) Inverse compactness of BSs is shown for the same solutions. The inset shows the maximal value of the scalar field along the BS lines.

mass, $R_{S c h w}=2 M_{99}$ :

$$
\text { Compactness }^{-1} \equiv \frac{R_{99}}{2 M_{99}} .
$$

The result for the inverse compactness of BSs with several values of $\xi$ is exhibited in Figure 1 (bottom panel). One can see that the inverse compactness is always greater than unity; in other words, BSs are less compact than BHs, as one would expect. Also, at least for the considered values, the non-minimal coupling $\xi \neq 0$ does not alter substantially the compactness of the BSs, tending to make such 
compactness smaller, for fixed frequency, in the strongest gravity region we have considered (towards the centre of the spiral).

Further insight on the influence of a nonzero coupling on the mass of the BS solutions can be found in Fig. 2, where the coupling constant $\xi$ is varied for three fixed values of the frequency $w$. Interestingly, while for a positive coupling, $M$ always increases with $\xi$ (for fixed frequency), a minimal mass value is approached for some negative $\xi$, with $M$ increasing with $|\xi|$ for lower $\xi$ values.

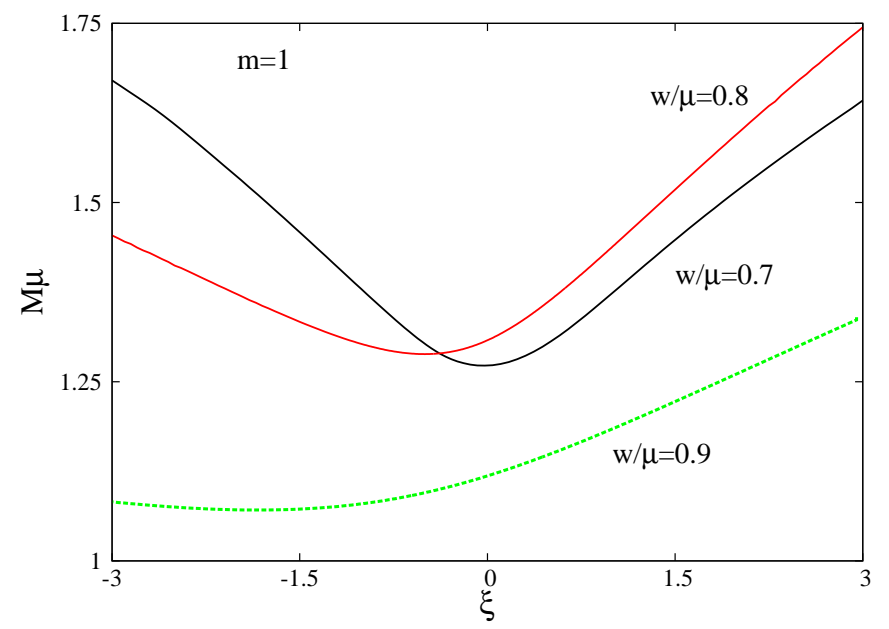

Fig. 2. The BSs mass is shown as a function of $\xi$ for three different frequencies.

\subsection{Black holes with synchronized scalar hair}

Before discussing the non-minimally coupled HBHs, let us recall that for $\xi=0$, the emergence of scalar hair can be seen in linearized theory, by considering the massive Klein-Gordon equation $\left(\nabla^{2}-\mu^{2}\right) \Psi=0$, as a test field, on a Kerr BH background. This was interpreted ${ }^{12}$ as a zero mode of the superradiant instability ${ }^{43}$ That is, by solving the Klein-Gordon equation on the Kerr background, real frequency bound states can be obtained when $w=m \Omega_{H}$, corresponding to linearized (hence nonbackreacting) hair, called stationary scalar clouds 12 44 47 However, since the Kerr BH solves the vacuum Einstein equations $(R=0)$, it is obvious that all results in the aforementioned works remain valid in the non-minimally coupled case. Thus one can predict that HBHs with $\xi \neq 0$ will branch off from the same set of Kerr $\mathrm{BHs}$ - forming the fundamental existence line $e^{12}$ - as the ones with $\xi=0$, a feature which is confirmed by our numerical solutions. However, when deviating from that line, the Ricci scalar deviates from zero and the effects of a non-zero coupling term become relevant.

A different path in constructing HBHs is to start instead with their solitonic 
limit. We have found that, for a given $\xi$, one can add a small $\mathrm{BH}$ at the center of any spinning BS, regardless of $w$. By increasing the horizon size from zero (via the parameter $r_{H}$ ), we obtain rotating $\mathrm{BH}$ solutions with $\Omega_{H}$ fixed by (30).
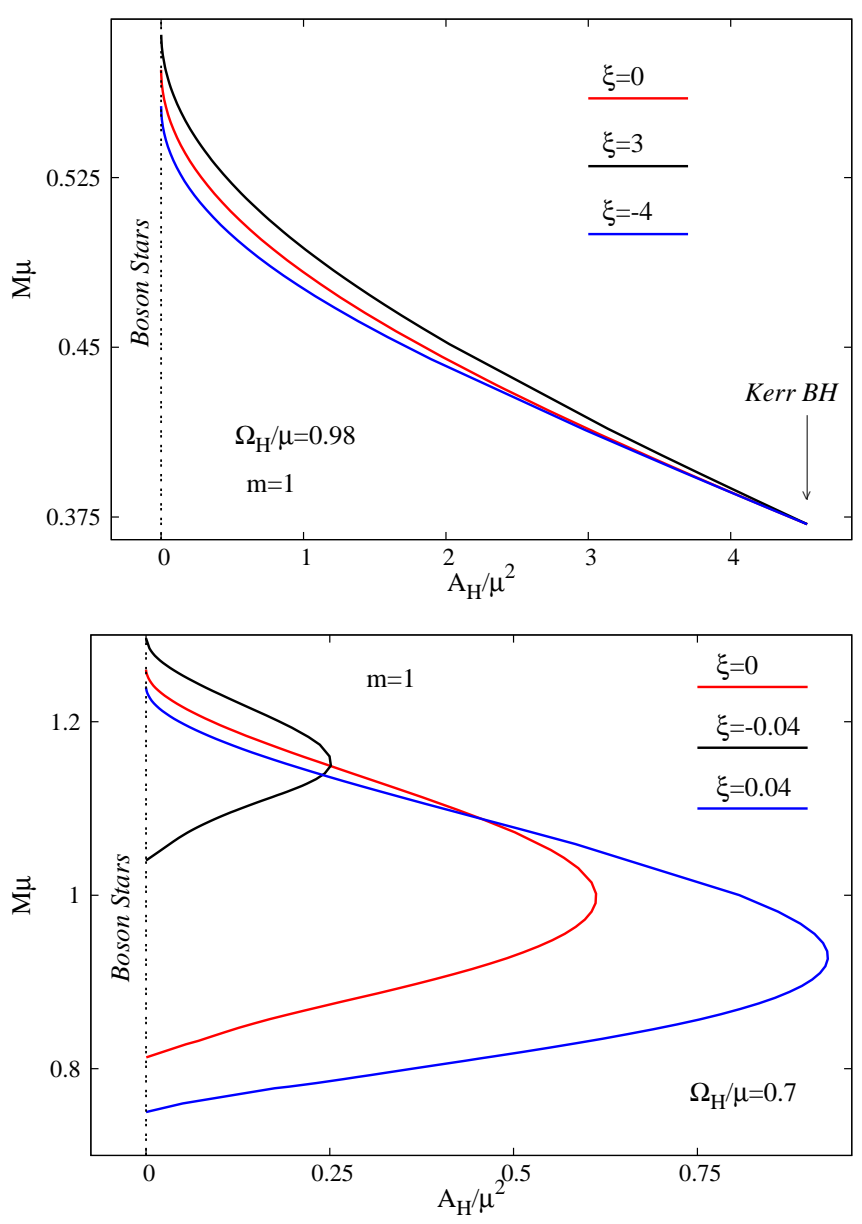

Fig. 3. Mass of the hairy BHs, in units of the scalar field mass, vs. the horizon area for three sets of solutions with the same angular velocity and different values of the coupling constant $\xi$.

A systematic, thorough study of the non-minimally coupled HBH solutions is beyond the scope of this work. Instead, in order to establish their existence and probe some basic properties, we have considered several values of $\xi$ and a set of frequencies with $w_{\min }<w<\mu$. In each case, we have studied HBHs for all allowed range of the $r_{H}$. The numerical results strongly suggest that all basic properties of the minimally coupled HBHs are kept. For any $\xi$, the region where HBHs exist is 
delimited by: $(i)$ the BS curve where the horizon shrinks to zero size, $(i i)$ the subset of Kerr solutions that support the fundamental existence line of stationary scalar clouds, and (iii) a set of extremal (i.e. zero temperature) HBHs.

Some results of the numerical integration are exhibited in Fig. 3 where we show the mass $M$ as a function of horizon area $A_{H}$ for several sets of HBHs solutions with fixed values of $\Omega_{H}$ (or, equivalently, $w$ ).

For a large enough frequency - e.g. $w / m=0.98$ in Fig. 3 (top panel) -, the HBHs interpolates between the corresponding BSs and the vacuum Kerr solution on the existence line. As $r_{H} \rightarrow 0$ the horizon area vanishes while the temperature diverges. For sufficiently small value $\mathrm{E}$ of $w / \mu$, the solutions interpolate between two BS solutions with the same scalar field frequency - e.g. $w / m=0.7$ in Fig. 3 (bottom panel).

Let us conclude with two final remarks. Firstly, for all solutions studied so far, the contribution of the non-minimal coupling term to the total entropy (14), was always several orders of magnitude smaller than the Einstein term. Secondly, that the bound for the horizon linear velocity $v_{H}, \frac{48}{,} v_{H}<1$, is fulfilled for all $\mathrm{HBH}$ solutions considered so far.

\section{Further remarks}

In this paper we have constructed rotating boson star solutions and initiated the study of hairy BHs in the EKG model with a non-minimal coupling of the scalar field to the Ricci tensor. One of the conclusions of our study is that the synchronisation mechanism to endow spinning BHs with hair ${ }^{51}$ survives yet another generalisation: considering a non-minimal coupling between the scalar field and the Ricci scalar. This adds up to the already extensive list of examples where this synchronisation mechanism allows the construction of hairy BHs, including: different matter fields (scalar ${ }^{12}[13 \sqrt[52]{52}$ and vector $\sqrt{53} \sqrt[54]{4}$ ), charged $\mathrm{BHs}, \sqrt[55]{5}$ in spacetime dimensions different than four,$\sqrt[56]{57}$ for non-asymptotically flat spacetimes,,$\sqrt[58]{ }$ with non-spherical horizon topologies ${ }^{59}$ and in scalar-tensor theories ${ }^{60}$

The physical picture concerning both the solitons and hairy BHs with nonminimal coupling is qualitatively similar to that obtained in the minimally coupled case. Two interesting technical aspects that we would like to emphasise are: 1) that the contribution of the non-minimal coupling to the ADM mass and angular momentum of a boson star, $M_{(\xi)}, J_{(\xi)}, c f$. (11), turns out to vanish globally, albeit being non-zero locally, $c f$. eq. (12); 2) That an elegant form for the entropy in terms of an effective Newton's constant can be obtained, $c f$. (15). The latter observation has been known in the literature, 61 to the best of our knowledge, however, the transformation of the volume integrals (11) for $M_{(\xi)}, J_{(\xi)}$ into the surface integrals (12); has not been previously observed.

e For some intermediate frequencies, one finds also branches of HBHs with fixed $w$ staring in BSs and ending in extremal BHs with scalar hair. These limiting configurations have finite horizon size and global charges. 
We would like to close by remarking that the solutions we have presented herein allow us to obtain, easily, a new set of solutions of the non-minimally coupled EKG model, with a particular choice of potential. By using the conformal rescaling of the action (1):

$$
\bar{g}_{\mu \nu}=\Omega^{2} g_{\mu \nu}, \quad \Omega^{2}=1-16 \pi G \xi \Psi^{*} \Psi=\frac{G}{G_{e f f}},
$$

the model we have considered can be mapped to the Einstein frame, that is a minimally coupled scalar field theory to gravity. $\Omega^{2}>0$ has a clear physical meaning since it implies $G_{\text {eff }}>0$, a condition which is satisfied by all solutions reported in this work. The pairs of variables (metric $g_{a b}$ and scalar $\Psi$ ) defined originally constitute what is called a Jordan frame. Consider now the transformation

$$
d \bar{\Psi}=\frac{\sqrt{1-16 \pi G \xi(1-6 \xi) \Psi^{*} \Psi}}{1-16 \pi G \xi \Psi^{*} \Psi} d \Psi
$$

such that, in the redefined action

$$
S=\int d^{4} x \sqrt{-\bar{g}}\left[\frac{\bar{R}}{16 \pi G}-\frac{1}{2} \bar{g}^{a b}\left(\bar{\Psi}_{, a}^{*} \bar{\Psi}_{, b}+\bar{\Psi}_{, b}^{*} \bar{\Psi}_{, a}\right)-V(\bar{\Psi})\right],
$$

the scalar field $\bar{\psi}$ becomes minimally coupled to $\bar{R}$. One pays the price, however, that the new scalar potential becomes more complicated,

$$
V(\bar{\Psi})=\frac{\mu^{2} \Psi^{*} \Psi}{\left(1-16 \pi G \xi \Psi^{*} \Psi\right)^{2}},
$$

with $\Psi$ a function of $\bar{\Psi}$, via the transformation (35).

The new variables (metric $\bar{g}_{a b}$ and scalar $\bar{\Psi}$ ) are said to constitute an Einstein frame. The transformation given by eqs. (34, 35) therefore maps a solution of the field equations (2), (3) to a solution that extremizes (36). The transformation is independent of any assumption of symmetry and, in this sense, it is covariant; one can easily infer that the transformation is one-to-one in general.

Therefore, all spinning solutions of the initial model (11) are mapped to spinning BSs and HBHs of the Einstein frame model (36). We note that the mass, angular momentum, Hawking temperature, horizon angular velocity together with the synchronization condition are not affected by the transformation (34), (35) (we recall that $\Psi \rightarrow 0$ asymptotically), while the entropy together with the mass and angular momentum stored in the scalar field are different in the two frames.

Furthermore, the Weyl rescaling (34) helps us to rule out the existence of static, spherically symmetric BH solutions in the original model (1) as long as $\Omega^{2}>0$. This can be shown as follows. We start by supposing the existence of a static BH solution in the original Jordan frame. Since the transformation (34, 35) preserves symmetries and the structure of the light cone, this results in a $\mathrm{BH}$ solution in the Einstein frame. However, this contradicts a well known no-hair theorem, $\frac{49}{4}$ that applies for a scalar field minimally coupled with gravity. This theorem applies to scalar fields that may (but need not to) vary harmonically with time and may have an arbitrary 
positive semidefinite potential. One can easily show that the proof of this no-hair theorem still applies for the model (36), since, in particular, the potential $V(\bar{\psi})$ is positive semidefinite. Therefore we conclude that no $\mathrm{BH}$ solutions exist also in the original Jordan frame. The existence of the BBMB solution circumvents this argument since $\Omega^{2}$ is not positive everywhere outside the horizon. Thus, this solution violates a requirement we may call conformal regularity: that the conformal factor $\Omega^{2}$ is non negative outside the horizon.

\section{Acknowledgments}

C. H. and E. R. acknowledge funding from the FCT-IF programme. This project has received funding from the European Union's Horizon 2020 research and innovation programme under the H2020-MSCA-RISE-2015 Grant No. StronGrHEP-690904, the H2020-MSCA-RISE-2017 Grant No. FunFiCO-777740 and by the CIDMA project UID/MAT/04106/2013. The authors would also like to acknowledge networking support by the COST Action GWverse CA16104.

\section{References}

1. G. Aad et al. [ATLAS Collaboration], Phys. Lett. B 716 (2012) 1 arXiv:1207.7214 [hep-ex]];

S. Chatrchyan et al. [CMS Collaboration], Phys. Lett. B 716 (2012) 30 arXiv:1207.7235 [hep-ex]].

2. E. Berti et al., Class. Quant. Grav. 32 (2015) 243001 arXiv:1501.07274 [gr-qc]].

3. C. A. R. Herdeiro and E. Radu, Int. J. Mod. Phys. D 24 (2015) no.09, 1542014 arXiv:1504.08209 [gr-qc]].

4. F. E. Schunck and E. W. Mielke, Class. Quant. Grav. 20 (2003) R301 arXiv:0801.0307 [astro-ph]].

5. D. J. Kaup, Phys. Rev. 172 (1968) 1331; R. Ruffini and S. Bonazzola, Phys. Rev. 187 (1969) 1767.

6. S. L. Liebling and C. Palenzuela, Living Rev. Rel. 15 (2012) 6 arXiv:1202.5809 [gr-qc]].

7. F. H. Vincent, Z. Meliani, P. Grandclement, E. Gourgoulhon and O. Straub, Class. Quant. Grav. 33 (2016) no.10, 105015 arXiv:1510.04170 [gr-qc]].

8. V. Cardoso, E. Franzin and P. Pani, Phys. Rev. Lett. 116 (2016) no.17, 171101 Erratum: [Phys. Rev. Lett. 117 (2016) no.8, 089902] arXiv:1602.07309 [gr-qc]].

9. Z. Cao, A. Cardenas-Avendano, M. Zhou, C. Bambi, C. A. R. Herdeiro and E. Radu, JCAP 1610 (2016) no.10, 003 arXiv:1609.00901 [gr-qc]].

10. A. Surez, V. H. Robles and T. Matos, Astrophys. Space Sci. Proc. 38 (2014) 107 arXiv:1302.0903 [astro-ph.CO]].

11. L. Hui, J. P. Ostriker, S. Tremaine and E. Witten, Phys. Rev. D 95 (2017) no.4, 043541 arXiv:1610.08297 [astro-ph.CO]].

12. C. A. R. Herdeiro and E. Radu, Phys. Rev. Lett. 112 (2014) 221101 arXiv:1403.2757 $[\mathrm{gr}-\mathrm{qc}]]$.

13. C. Herdeiro and E. Radu, Class. Quant. Grav. 32 (2015) no.14, 144001 arXiv:1501.04319 [gr-qc]].

14. S. Capozziello and M. De Laurentis, Phys. Rept. 509 (2011) 167 arXiv:1108.6266 [gr-qc]].

15. V. Faraoni, Int. J. Theor. Phys. 40 (2001) 2259 hep-th/0009053. 
16. V. Faraoni, E. Gunzig and P. Nardone, Fund. Cosmic Phys. 20 (1999) 121 gr-qc/9811047.

17. N. D. Birrell and P. C. Davies, Quantum field in curved Space, Cambridge University Press, Cambridge, (1982).

18. T. Futamase and K. i. Maeda, Phys. Rev. D 39 (1989) 399.

19. J. D. Bekenstein, Annals Phys. 82 (1974) 535.

20. N. M. Bocharova, K. A. Bronnikov, V. N. Melnikov, Vestn. Mosk. Univ. Fiz. Astron. 6 (1970) 706.

21. A. E. Mayo and J. D. Bekenstein, Phys. Rev. D 54 (1996) 5059 gr-qc/9602057.

22. J. D. Bekenstein, in Moscow 1996, 2nd International A.D. Sakharov Conference on physics, 216-219 gr-qc/9605059.

23. S. Hod, Phys. Rev. D 96 (2017) no.12, 124037.

24. S. Hod, Phys. Lett. B 771 (2017) 521.

25. C. Barcelo and M. Visser, Phys. Lett. B 466 (1999) 127 gr-qc/9908029.

26. C. Barcelo and M. Visser, Class. Quant. Grav. 17 (2000) 3843 [gr-qc/0003025].

27. J. J. van der Bij and E. Radu, Nucl. Phys. B 585 (2000) 637 hep-th/0003073.

28. A. V. Nguyen and K. C. Wali, Phys. Rev. D 51 (1995) 1664 hep-ph/9310370.

29. Y. Brihaye and Y. Verbin, Phys. Rev. D 91 (2015) no.6, 064021 arXiv:1412.0478 $[\mathrm{gr}-\mathrm{qc}]]$.

30. J. J. van der Bij and M. Gleiser, Phys. Lett. B 194 (1987) 482.

31. C. Herdeiro and E. Radu, Phys. Rev. D 89 (2014) no.12, 124018 arXiv:1406.1225 [gr-qc]].

32. C. A. R. Herdeiro, E. Radu and H. F. Rnarsson, Int. J. Mod. Phys. D 25 (2016) no.09, 1641014 arXiv:1604.06202 [gr-qc]].

33. D. Horvat and A. Marunovi, Class. Quant. Grav. 30 (2013) 145006 arXiv:1212.3781 [gr-qc]].

34. D. Horvat, S. Ilijic, A. Kirin and Z. Narancic, Class. Quant. Grav. 30 (2013) 095014 arXiv:1302.4369 [gr-qc]].

35. S. Yoshida and Y. Eriguchi, Phys. Rev. D 56 (1997) 762.

36. D. Garfinkle, G. T. Horowitz and A. Strominger, Phys. Rev. D 43 (1991) 3140 Erratum: [Phys. Rev. D 45 (1992) 3888].

37. J. M. Bardeen, B. Carter and S. W. Hawking, Commun. Math. Phys. 31 (1973) 161.

38. P. K. Townsend, gr-qc/9707012.

39. R. M. Wald, Phys. Rev. D 48 (1993) no.8, R3427 gr-qc/9307038.

40. V. Iyer and R. M. Wald, Phys. Rev. D 50 (1994) 846 gr-qc/9403028.

41. F. E. Schunck and E. W. Mielke, Phys. Lett. A 249 (1998) 389.

42. P. Grandclement, C. Som and E. Gourgoulhon, Phys. Rev. D 90 (2014) no.2, 024068 arXiv:1405.4837 [gr-qc]].

43. R. Brito, V. Cardoso and P. Pani, Lect. Notes Phys. 906 (2015) pp.1 arXiv:1501.06570 [gr-qc]].

44. S. Hod, Phys. Rev. D 86 (2012) 104026 Erratum: [Phys. Rev. D 86 (2012) 129902] arXiv:1211.3202 [gr-qc]].

45. S. Hod, Eur. Phys. J. C 73 (2013) no.4, 2378 arXiv:1311.5298 [gr-qc]].

46. S. Hod, Phys. Rev. D 90 (2014) no.2, 024051 arXiv:1406.1179 [gr-qc]].

47. C. L. Benone, L. C. B. Crispino, C. Herdeiro and E. Radu, Phys. Rev. D 90 (2014) no.10, 104024 arXiv:1409.1593 [gr-qc]].

48. C. A. R. Herdeiro and E. Radu, Int. J. Mod. Phys. D 24 (2015) no.12, 1544022 arXiv:1505.04189 [gr-qc]].

49. I. Pena and D. Sudarsky, Class. Quant. Grav. 14 (1997) 3131.

50. P. Amaro-Seoane, J. Barranco, A. Bernal and L. Rezzolla, JCAP 1011 (2010) 002 

arXiv:1009.0019 [astro-ph.CO]].

51. C. A. R. Herdeiro and E. Radu, Int. J. Mod. Phys. D 23 (2014) no.12, 1442014 arXiv:1405.3696 [gr-qc]].

52. C. A. R. Herdeiro, E. Radu and H. Rnarsson, Phys. Rev. D 92 (2015) no.8, 084059 arXiv:1509.02923 [gr-qc]].

53. C. Herdeiro, E. Radu and H. Runarsson, Class. Quant. Grav. 33 (2016) no.15, 154001 arXiv:1603.02687 [gr-qc]].

54. C. A. R. Herdeiro and E. Radu, Phys. Rev. Lett. 119 (2017) no.26, 261101 arXiv:1706.06597 [gr-qc]].

55. J. F. M. Delgado, C. A. R. Herdeiro, E. Radu and H. Runarsson, Phys. Lett. B 761 (2016) 234 arXiv:1608.00631 [gr-qc]].

56. Y. Brihaye, C. Herdeiro and E. Radu, Phys. Lett. B 739 (2014) 1 arXiv:1408.5581 [gr-qc]].

57. C. Herdeiro, J. Kunz, E. Radu and B. Subagyo, Phys. Lett. B 748 (2015) 30 arXiv:1505.02407 [gr-qc]].

58. O. J. C. Dias, G. T. Horowitz and J. E. Santos, JHEP 1107 (2011) 115 arXiv:1105.4167[hep-th]].

59. C. Herdeiro, J. Kunz, E. Radu and B. Subagyo, Phys. Lett. B 779 (2018) 151 arXiv:1712.04286 [gr-qc]].

60. B. Kleihaus, J. Kunz and S. Yazadjiev, Phys. Lett. B 744 (2015) 406 arXiv:1503.01672 [gr-qc]].

61. A. Ashtekar, A. Corichi and D. Sudarsky, Class. Quant. Grav. 20 (2003) 3413 gr-qc/0305044. 